\title{
DENSE SUBSETS OF $\beta X$
}

\section{R. LEVY AND R. H. MCDOWELL}

ABSTRACT. An easy lemma is proved which assures the existence of certain dense subsets of $\beta X$ on the basis of the existence of similar dense subsets of any compactification of $X$.

1. Introduction. In this paper, we show that, using an easily established property of the Stone extension of a mapping, it is possible to find dense sets in the Stone-Čech compactification $\beta X$ of a space corresponding to dense sets in any other compactification $X^{*}$. Using this device, we obtain several new results, and some greatly simplified proofs of known results.

For example, we show that if $X$ is a separable metric space, then every dense subset of $\beta X$ is separable. We also show that $\beta X$ is separable if and only if $X$ has any separable compactification, and use this result to obtain a nonseparable space $X$ such that $\beta X$ is separable. In addition, we obtain conditions ensuring that $\beta X$ has the Blumberg property.

2. The basic lemma. All given spaces are assumed to be completely regular and Hausdorff. If $X$ is any such space, we denote its Stone-Čech compactification, as usual, by $\beta X$. If $X^{*}$ is any compactification of $X$, $i^{*}: \beta X \rightarrow X^{*}$ will denote the (unique) continuous extension from $\beta X$ onto $X^{*}$ of the identity map $i: X \rightarrow X$.

2.1. Lemma. Let $X^{*}$ be any compactification of $X$. Then a set $D$ is dense in $\beta X$ if and only if $i^{*}(D)$ is dense in $X^{*}$.

Proof. Suppose $i^{*}(D)$ is dense in $X^{*}$. Then $i^{*}\left(\mathrm{cl}_{\beta X} D\right)$ is a compact subset of $X^{*}$ containing a dense subset, and is hence all of $X^{*}$. It follows that $X \subset \mathrm{cl}_{\beta X} D$ and, hence, $\mathrm{cl}_{\beta X} D=\beta X$; i.e., $D$ is dense in $\beta X$.

The converse is immediate.

Received by the editors March 20, 1974.

AMS (MOS) subject classifications (1970). Primary 54D35; Secondary 54A25, $54 \mathrm{C} 30$.

Key words and phrases. Dense-separable, Stone-Čech compactification, Blumberg's theorem. 
3. Dense-separable spaces. A space is called dense-separable if every dense subset is separable.

3.1. Theorem. The following conditions on $X$ are equivalent:

(i) $\beta X$ is dense-separable.

(ii) Every compactification of $X$ is dense-separable.

(iii) $X$ bas a dense-separable compactification.

Proof. (i) implies (ii). Let $D$ be a dense subset of a compactification $X^{*}$ of $X$. By Lemma 2.1, $i^{*-1}(D)$ is a dense subset of $\beta X$, which, by (i), contains a countable dense subset $\tilde{A}$. Now $i^{*}(\tilde{A})$ is a countable dense subset of $D$.

(ii) implies (iii) is obvious.

(iii) implies (i). Let $X^{*}$ be any dense-separable compactification of $X$, and suppose $D$ is a dense subset of $\beta X$. Then $i^{*}(D)$ is dense in $X^{*}$; let $A$ be a countable dense subset of $i^{*}(D)$. Let $\widetilde{A}$ be a countable subset of $D$ that maps onto $A$. Then by Lemma $2.1, \widetilde{A}$ is dense in $\beta X$, and hence in $D$.

3.2. Corollary. $\beta X$ is dense-separable if $X$ is either (1) separable metric or (2) separable and ordered.

Proof. (1) A separable metric space has a metric (hence dense-separable) compactification. Hence $\beta X$ is dense-separable, by Theorem 3.1. (2) If $X$ is a separable ordered space, then $X$ has an ordered (separable) compactification. But a separable ordered space is hereditarily separable (see [6]). Thus, $X$ has a dense-separable compactification, so $\beta X$ is dense-separable.

The following theorem is proved the same way as Theorem 3.1.

3.3. Theorem. The following conditions on $X$ are equivalent:

(i) $\beta X$ is separable.

(ii) Every compactification of $X$ is separable.

(iii) $X$ bas a separable compactification.

4. Blumberg spaces. A space $X$ is called a Blumberg space if for every real valued function $f$ defined on $X$, there is a dense subset $D$ of $X$ such that $f$ restricted to $D$ is continuous. It was shown in [1] that a metric space is Blumberg if and only if it is a Baire space. Recently, it has been shown [5] that it is consistent with the usual axioms for set theory, including the axiom of choice, that there exist a compact Hausdorff 
space that is not Blumberg. The next theorem gives a useful condition on $X$ ensuring that $\beta X$ is Blumberg.

4.1. Theorem. If $X$ is dense in any Blumberg space, then $\beta X$ is Blumberg. In particular, $\beta X$ is Blumberg if $X$ is metrizable.

Proof. Suppose $X$ is dense in the Blumberg space $T$, and let $X^{*}$ be any compactification of $T$. Clearly, $X^{*}$ is also Blumberg. Now let $f$ be any real valued function on $\beta X$. For each $p \in X^{*}$, choose a point $x_{p} \epsilon$ $i^{*-1}(p)$, and define a real valued function $g$ on $X^{*}$ by setting $g(p)=f\left(x_{p}\right)$. Since $X^{*}$ is Blumberg, there is a dense subset $A$ of $X^{*}$, such that $g$ restricted to $A$ is continuous. Let $\widetilde{A}=\left\{x_{p}: p \in A\right\}$. Then by Lemma $2.1, \tilde{A}$ is dense in $\beta X$. Further, $f$ is continuous on $\tilde{A}$, since there it agrees with the continuous function $g \circ i^{*}$.

Finally, if $X$ is metrizable, $X$ has a metric completion, which is Blumberg [1]. Hence $\beta X$ is Blumberg. (This last result was also obtained by H. E. White [7], using different methods.)

Remark. In [4] an example is given of a space which can be proved to be Blumberg by Theorem 4.1 but not by any other known method.

4.2. Lemma. Let $N$ be a countably infinite discrete space. A topological space $X$ is a Baire space if and only if, for each $f: X \rightarrow N$, there is a dense subset $D$ of $X$ such that $f$ restricted to $D$ is continuous.

A proof can be found in [4] or [7]. Using this lemma, we can prove the following theorem in the same way as we proved Theorem 4.1.

4.3. Theorem. If the space $X$ bas a compactification $X^{*}$ such that every dense subset of $X^{*}$ is Baire, then every dense subset of $\beta X$ is Baire.

5. Examples. We denote the real numbers by $R$, the rationals by $Q$, and the integers by $N$.

5.1. Example (see also [2]). By Corollary 3.2, $\beta Q$ is dense-separable. It follows that $\beta Q \backslash Q$ is dense-separable. Also, since $\beta Q$ is a dense-separable compactification of $\beta Q \backslash Q, \beta(\beta Q \backslash Q)$ is dense-separable.

5.2. Example. By Theorem 4.1, $\beta Q$ is Blumberg. We now show that $\beta Q \backslash Q$ is Blumberg. Let $f$ be any real function on $\beta Q \backslash Q$, and $\left\{r_{f}\right\}$ an enumeration of $Q$. Define $\tilde{f}: \beta Q \rightarrow R$ as the extension of $f$ obtained by setting $\tilde{f}\left(r_{i}\right)=i$ for each $i$. Since $\beta Q$ is Blumberg, $\tilde{f}$ is continuous on 
a dense subset $D$ of $\beta Q$. Since $D \cap Q$ is nowhere dense, $D \cap(\beta Q \backslash Q)$ is dense in $\beta Q \backslash Q$. Thus $f$ restricted to this dense subset of $\beta Q \backslash Q$ is continuous.

For the next two examples, see [3] for a different proof.

5.3. Example. A separable, compact space that is not dense-separable.

Let $I$ be the closed unit interval, and let $X=I^{I}$, furnished with the product topology. It is well known that $X$ is separable. Let $Y=\{f \in X$ : $f(x) \neq 0$ for only finitely many values of $x\}$. Now $Y$ is easily seen to be dense in $X$. But $Y$ is not separable. To see this, let $\left\{f_{i}\right\}$ be any countable subset of $Y$, and, for each $i$, set $F_{i}=\left\{x \in I: f_{i}(x) \neq 0\right\}$. Since $F_{i}$ is finite for each $i, F=\bigcup_{i} F_{i}$ is countable. Choose an $x_{0} \in I \backslash F$, and let $U=$ $\left\{f \in Y: f\left(x_{0}\right)>1 / 2\right\}$. Then $U$ is a nonempty open set in $Y$ containing no $f_{i}$, so that $\left\{f_{i}\right\}$ is not dense in $Y$. Thus, $X$ is separable but not dense-separable.

5.4. Example. A nonseparable space with separable Stone-Čech compactification.

The space is the space $Y$ of the previous example. Since $Y$ has a separable compactification, namely $X$, it follows from Theorem 3.3 that $\beta Y$ is separable.

5.5. Example. For each infinite cardinal $y \leq 2{ }^{\boldsymbol{N}_{0}}$, we construct a separable space that is a union of $\gamma$ pairwise disjoint copies of $\beta N \backslash N$.

Let $A$ be a set of cardinal $\gamma$, and let $\left\{x_{\alpha}: \alpha \in A\right\}$ be a dense subset of $R \backslash Q$ such that $x_{\alpha} \neq x_{\alpha^{\prime}}$ if $\alpha \neq \alpha^{\prime}$. For each $\alpha$, let $s_{\alpha}$ be a sequence in $Q$ converging to $x_{a}$. Then if $\alpha \neq \alpha^{\prime}, s_{\alpha} \cap s_{\alpha^{\prime}}$ is finite and each $s_{a}$ is a countably infinite discrete $C^{*}$-embedded subset of $Q$. Therefore, $X=$ $\bigcup_{a \in A}\left(\mathrm{cl}_{\beta Q^{S}} \backslash Q\right)$ is a union of $\gamma$ pairwise disjoint copies of $\beta N \backslash N$. Further, since $X$ is dense in $\beta Q, X$ is separable (Example 5.1). In fact, since $X$ has a dense-separable compactification, namely $\beta Q, \beta X$ is dense-separable. $\boldsymbol{\aleph}_{0}$ Question. Is there a separable space which is the union of more than
pairwise disjoint copies of $\beta N \backslash N$ ?

\section{REFERENCES}

1. J. C. Bradford and C. Goffman, Metric spaces in which Blumberg's theorem holds, Proc. Amer. Math. Soc. 11 (1960), 667-670. MR 26 \#3832.

2. W. W. Comfort, Addendum to a paper of J. de Groot, Bull. Acad. Polon. Sci. Sér. Sci. Math. Astronom. Phys. 17 (1969), 361-362. MR 41 \#9204.

3. - An example in density character, Arch. Math. 14 (1963), 422-423. MR 28 \# 4503. 
4. R. Levy, Non-Blumberg Eaire spaces and related topics, Thesis, Washington University, 1974.

5. - Strongly non-Blumberg spaces, General Topology and Appl. 4 (1974), 173-177.

6. D. J. Lutzer and H. R. Bennett, Separability, the countable chain condition and the Lindelöf property in linearly orderable spaces, Proc. Amer. Math. Soc. 23 (1969), 664-667. MR 40 \#2013.

7. H. E. White, Blumberg's theorem in topological spaces (preprint).

DEPARTMENT OF MATHEMATICS, WASHINGTON UNIVERSITY, ST. LOUIS, MISSOURI 63130 (Current address of R. H. McDowell)

Current address (R. Levy): Department of Mathematics, Goucher College, Towson, Maryland 21228 